\title{
Effect of Changes in Coronary Arterial Blood Flow on the Myocardial Contractile Force
}

\author{
Jiro Nakano, M.D.
}

The direct effect of changes in coronary arterial blood flow on the myocardial contractile force was studied in anesthetized dogs. Coronary blood flow in the anterior descending branch was changed stepwise by producing different degrees of coronary constriction or by using a Sigmamotor pump. Myocardial contractile force was measured using WaltonBrodie strain gauge arches. It was found that the stepwise decreases in coronary arterial blood flow reduced stepwise myocardial contractile force of the ischemic portion of the ventricle. On the other hand, the supernormal increase in coronary arterial blood flow caused no essential change in the contractile force. The coronary arterial ligation caused the maximum decrease in contractile force in the injured portion of the ventricle. In contrast, the ligation usually increased contractile force in the healthy portion of the ventricle. This is most likely due to the reflex increase in the sympathoadrenal activity.

$\mathrm{M}$ ANY investigators ${ }^{1-5)}$ have found that experimentally induced coronary arterial occlusion results in evolutional electrophysiological changes of the heart. Orias ${ }^{6}$ observed that coronary arterial occlusion reduced ventricular systolic pressure as well as the speed of the pressure rise. Later, Tennant and Wiggers ${ }^{7}$ showed that coronary arterial ligation causes progressive enfeeblement of myocardial contraction. In addition, many investigators ${ }^{8)}$ found that myocardial hypoxia decreases myocardial contents of adenosine triphosphate (ATP) and creatine phosphate (CP) and consequently reduces contractile force in the isolated hearts of various species of animals. However, apparently, no systematic study has been made in regard to the relationship between the magnitude of coronary arterial blood flow and myocardial contractile force in animals with intact circulation. The current study was undertaken to investigate the direct effect of changes in coronary arterial blood flow on myocardial contractile force in anesthetized dogs.

From the Department of Pharmacology, University of Oklahoma School of Medicine, Oklahoma City, Oklahoma, U.S.A.

Received for publication October 13, 1965.

This work was supported by research grants (HE 07334 and HE 08057) from the U.S. Public Health Services. 


\section{Methods}

Twenty-seven dogs weighing between 21.5 and $28.0 \mathrm{Kg}$. were anesthetized with the intravenous administration of sodium pentobarbital $(30 \mathrm{mg} . / \mathrm{Kg}$.) or chloralose $(100 \mathrm{mg} . / \mathrm{Kg}$ ). The left hemithorax was opened under artificial respiration with a Palmer pump. In all experiments, the blood was rendered incoagulable by the intravenous administration of $5.0 \mathrm{mg}$. $/ \mathrm{Kg}$. of heparin sodium, followed by 2.5 $\mathrm{mg} . / \mathrm{Kg}$. every subsequent half hour. The methods used to study the effect of graded changes in coronary arterial blood flow on the myocardial contractile force are depicted in Fig. 1 and 2. A segment of the anterior descending branch of the left coronary artery was dissected. Immediately (usually less than $30 \mathrm{sec}$.) after the ligation of the dissected artery, the distal anterior descending branch was cannulated and perfused spontaneously or artificially using the following two techniques. As depicted in Fig. 1, in 8 dogs, the blood was allowed to flow spontaneously in the coronary artery via the left subclavian artery through a Shipley-Wilson rotameter, ${ }^{4)}$ with which the coronary arterial blood flow was measured continuously. A screw clamp was placed on the outflow tygon tubing of the rotameter to induce desired degrees of coronary constriction. As shown in Fig. 2, in additional 13 dogs the distal anterior descending branch was perfused at known and constant rates with blood shunted from the left subclavian artery using a Sigmamotor pump (Model TM2). The flow rate of the pump was calibrated before and after each experiment, and was found to be stable throughout the experimental period.

In all experiments, mean systemic and coronary arterial perfusion pressures were measured continuously with Statham pressure transducers (P23AA). In some

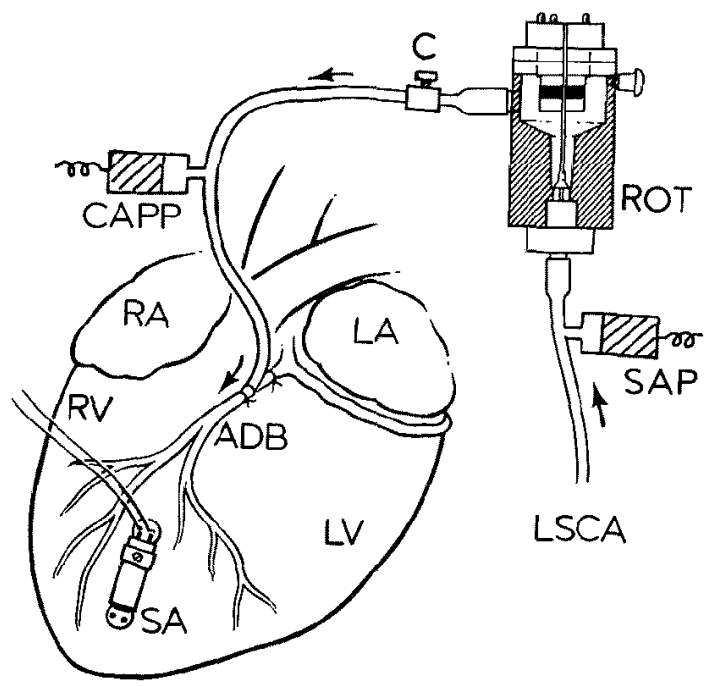

Fig. 1. The first method used to study the effect of graded coronary blood flows on myocardial contractile force. RA, LA, RV, LV, ADB and LSCA denote, respectively, right atrium, left atrium, right ventricle, left ventricle, anterior descending branch and left subclavian artery. SA, CAPP, C, ROT and SAP denote, respectively, a Walton-Brodie strain gauge arch, mean coronary arterial perfusion pressure, a screw clamp, a Shipley-Wilson rotameter and mean systemic arterial pressure. 


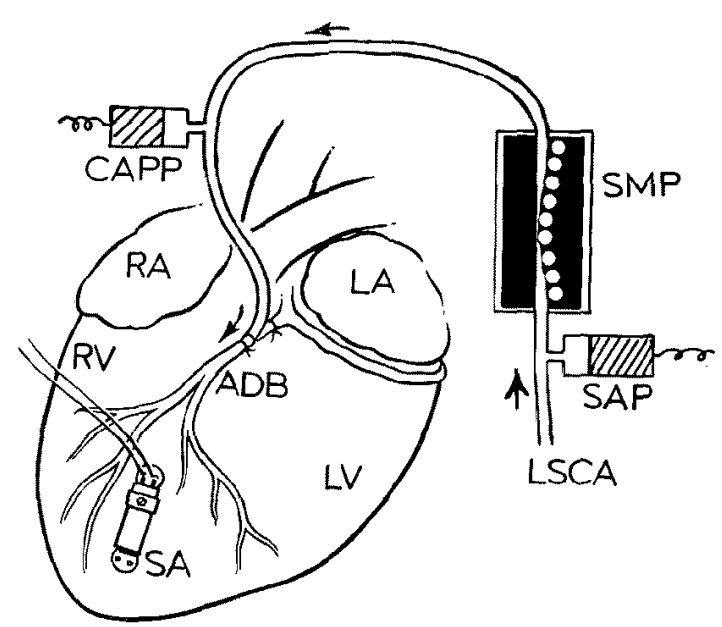

Fig. 2. The second method used to study the effect of graded coronary blood flow on myocardial contractile force. SMP denotes a Sigmamotor pump.

experiments, mean left atrial pressure was measured continuously with a Statham pressure transducer (P23B) connected to a catheter placed in the left atrium through a pulmonary vein. Heart rate and myocardial contractile force were continuously measured, respectively, by an Electronics for Medicine (EFM) tachometer and by a Walton-Brodie strain gauge arch. ${ }^{10), 11)}$ The arch was sutured to the anterior surface of the left ventricle near the apex which was perfused by the cannulated anterior descending branch. In 6 dogs, an additional strain gauge arch was sutured to the lateral-basal surface of the left ventricle which was supplied by coronary arteries other than the anterior descending branch. The area of the distribution of the anterior descending branch of the left coronary artery was delineated after each experiment by injecting indigo carmine into the artery. All hemodynamic parameters except heart rate were recorded continuously with an EFM research recorder (DR8). The coronary arterial peripheral resistance was calculated from the following formula: Coronary arterial peripheral resistance $(\mathrm{mm} . \mathrm{Hg} / \mathrm{ml} . / \mathrm{min})=$. mean coronary arterial perfusion pressure $(\mathrm{mm} . \mathrm{Hg}) /$ mean coronary arterial blood flow (ml./min.).

\section{REsults}

1. Hemodynamic effects of partial coronary constriction.

The effects of graded degree of coronary arterial constriction on heart rate, mean systemic arterial pressure, coronary arterial blood flow and mean coronary arterial perfusion pressure were studied in 8 dogs. The results were consistent in all experiments. A tracing of a representative experiment is illustrated in Fig. 3. A sudden partial constriction of the anterior descending branch caused immediate decreases in coronary arterial blood flow and mean coronary arterial perfusion pressure without any change in heart rate and mean 
systemic arterial pressure (Fig. 3A). On the other hand, myocardial contractile force decreased gradually and progressively. Shortly after its sudden decrease, the coronary arterial flow began increasing progressively as the coronary perfusion pressure decreased gradually. Approximately 1 to $2 \mathrm{~min}$. after the beginning of the coronary constriction, both myocardial contractile force and coronary arterial flow stabilized at levels significantly below each control. On cessation of the coronary occlusion, the perfusion pressure returned studdenly to control, whereas the coronary arterial flow increased markedly above control before its rather rapid recovery. On the other hand, myocardial contractile force recovered gradually to control (Fig. 3A). As seen in Fig. $3 \mathrm{~B}$, a greater degree of coronary constriction caused greater decreases in coronary arterial blood flow, coronary arterial perfusion pressure and myocardial contractile force. In addition, mean systemic arterial pressure also decreased gradually. With this degree of coronary constriction, myocardial contractile force decreased progressively and heart rate increased slightly. The coronary arterial flow did not recover although coronary perfusion pressure decreased progressively. On cessation of the constriction, the coronary arterial flow increased suddenly and more markedly above control, and returned more slowly than that observed with milder constriction. The coronary perfusion pressure rose suddenly toward control, and then recovered slowly as mean systemic arterial pressure and myocardial contractile force returned slowly to controls.

2. Hemodynamic effects of graded changes in coronary arterial blood

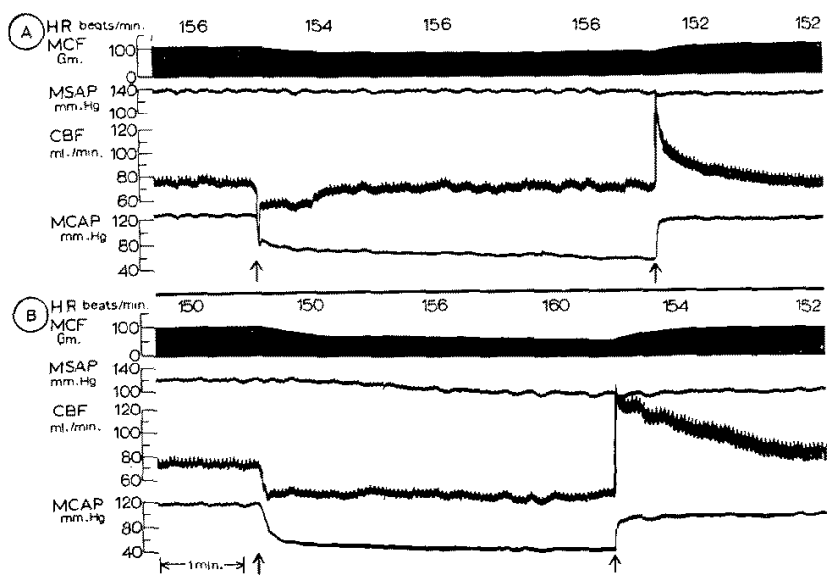

Fig. 3. Effects of mild (upper tracing) and severe (lower tracing) degrees of coronary constriction on heart rate (HR), myocardial contractile force (MCF), mean systemic arterial pressure (MSAP), mean coronary arterial blood flow (CBF) and mean coronary arterial perfusion pressure (MCAP) in a dog. The partial coronary constrictions were produced between the verticle arrows. 
flow using a Sigmamotor pump.

The effects of graded coronary arterial flow on heart rate, mean systemic arterial pressure, mean coronary arterial perfusion pressure, coronary arterial peripheral resistance, mean left atrial pressure and myocardial contractile force were studied in 13 dogs. The summary of the average hemodynamic effects is illustrated in Fig. 4. Initially, coronary arterial perfusion flow was set arbitrarily at a rate of $75 \mathrm{ml} . / \mathrm{min}$., and all hemodynamic parameters were observed to stabilize for as least $20 \mathrm{~min}$. Thereafter, coronary arterial blood flow was increased and decrcased evcry $15 \mathrm{~min}$. by $25 \mathrm{ml} . / \mathrm{min}$. using a Sigmamotor. The hemodynamic parameters were determined at the end of each increment or decrement. As seen in Fig. 4, stepwise decreases in coronary arterial flow caused stepwies decreases in coronary perfusion pressure and myocardial contractile force, and stepwise increase in coronary peripheral resistance. Throughout the experimental period, heart rate, systemic arterial pressure and left atrial pressure remained essentially unchanged. On the other hand, progressive increase in coronary arterial flow caused essentially no change in heart rate, systemic arterial pressure, left atrial pressure and myocardial contractile force. However, stepwise increase in coronary flow caused stepwise increase in coronary perfusion pressure and stepwise decrease in coronary peripheral resistance.

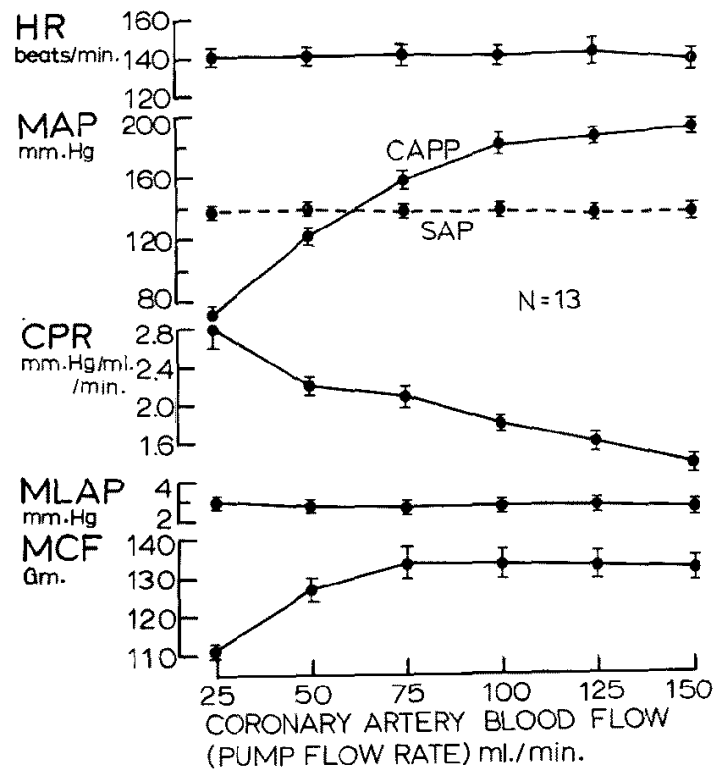

Fig. 4. Summary of average effects of graded coronary blood flow on heart rate, mean systemic arterial pressure, mean coronary arterial perfusion pressure, coronary arterial peripheral resistance (CPR), mean left arterial pressure and myocardial contractile force in 13 dogs. 


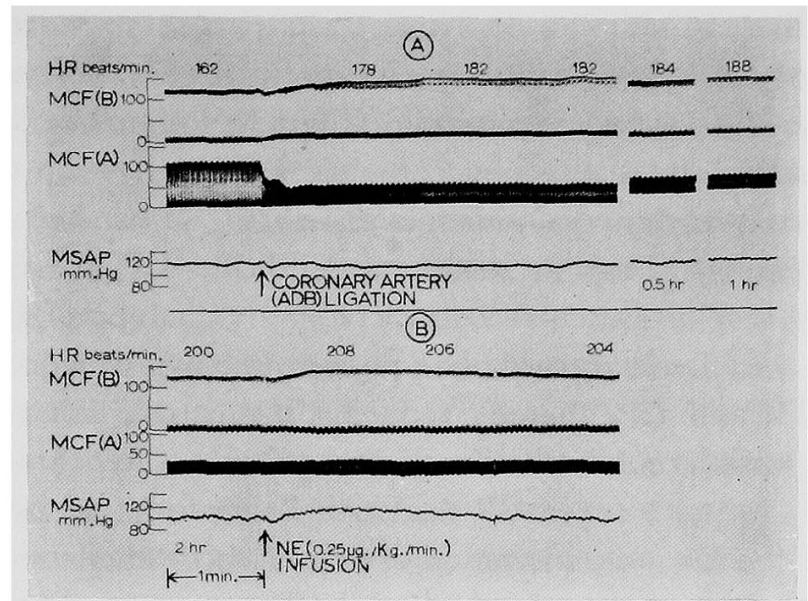

Fig. 5. Effects of the ligation of the anterior descending branch (ADB) of the left coronary artery on heart rate, myocardial contractile forces of the left ventricular base (B) and apex (A), and mean systemic arterial pressure. In the lower tracing, continuous infusion of norepinephrine (NE) was started approximately 2 hours after the ligation. The base lines of both myocardial contractile forces were lowered between the upper and lower tracings.

3. Hemodynamic effects of coronary arterial ligation, and of subsequent infusion of norepinephrine.

The effects of the coronary artery ligation on the hemodynamic parameters were studied in 6 dogs. A tracing of a representative experiment is illustrated in Fig. 5. A sudden ligation of the artery caused a marked decrease in myocardial contractile force of the left ventricular apex perfused by the artery (Fig. 5A). On the other hand, myocardial contractile force of the left ventricular base, which was not perfused by the anterior descending branch, increased gradually and significantly, as systemic arterial pressure decreased slightly. Approximately 2 hours after the ligation, continuous intravenous infusion of norepinephrine $(0.25 \mu \mathrm{g} . / \mathrm{Kg} . / \mathrm{min}$.) increased heart rate, myocardial contractile force of the left ventricular base and systemic arterial pressure without any significant change in the contractile force of the injured ventricle (left ventricular apex) (Fig. 5B).

\section{Discussion}

From the present study, it seems evident that stepwise decrease in coronary arterial blood flow per se causes stepwise reduction in myocardial contractile force. This observation is essentially in accordance with the previous report made by Tennant and Wiggers, ${ }^{7)}$ that coronary arterial ligation results progressively in hypodynamic ventricular contraction. When the infarcted ven- 
tricle is extensive, it may lower systolic ventricular pressure and systemic pressure, and may also decrease the maximum rate of development of isometric tension because of secondary myocardial failure. ${ }^{61,12), 13}$ ) The decreased myocardial contractile force caused by coronary arterial ligation or constriction is most likely due to myocardial ischemia or injury. It has been demonstrated that the high energy phosphates such as ATP and CP are essential to the biochemical process of muscular contraction. ${ }^{14)}$ Chang, ${ }^{15)}$ Michal et al.. ${ }^{16)}$ and Furchgott and $\mathrm{Lee}^{\mathrm{B}}$ showed that myocardial hypoxia decreases rapidly myocardial ATP and CP, thereby reducing myocardial contractile force essentially in proportion to the duration and magnitude of hypoxia. Myocardial hypoxia usually causes a rapid CP depletion being followed by a progressive decrease in ATP. The administration of oxygen to the ischemic myocardium results in rather slow recovery in ATP and GP contents. The present study also showed that the sudden coronary constriction decreases gradually and progressively myocardial contractile force, suggesting the above mentioned biochemical process. In contrast, a sudden release of the constriction causes gradual decrease in contractile force which also suggests the gradual recovery of ATP and CP in the myocardium. The degree of existing myocardial hypoxia is usually reflected by the magnitude of reactive hyperemia, ${ }^{17)}$ and the speed of recovery of the contractile force following reoxygenation. Green and Wegria ${ }^{18)}$ showed that myocardial ischemia is one of the most effective factors for coronary dilatation. Hence, the inception of a given coronary constriction always results in a marked coronary dilatation peripheral to the site of occlusion or constriction. This compensatory mechanism is demonstrated clearly in the experiment shown in Fig. 3A. Coronary arterial blood flow fell precipitously upon the coronary constriction, and then recovered markedly toward control as the coronary peripheral pressure decreased progressively. This compensatory mechanism would tend to increase myocardial contractile force to some degree, but usually could not overcome completely the initial reduction in oxygen tension which decreases ATP and CP. Apparently, a biochemical, physiological steady state is attained during mild coronary constriction and thus, after its initial decrease, myocardial contractile force stabilizes at a certain level. In contrast, with more marked coronary constriction, the decrease in peripheral pressure usually is not sufficient to increase coronary arterial flow because systemic arterial pressure also decreases progressively due to more extensive and severe myocardial failure. ${ }^{6)}$ Obviously, from Poiseuillc's law, the gradient between systemic arterial pressure and peripheral coronary pressure determines coronary arterial flow over a given resistance (the site of the coronary constriction). Hence, as systemic pressure and subsequently coronary arterial flow decrease further, myocardial contractile force decreases 
progressively in severe degrees of coronary constriction (Fig. 3B).

In the present study, the supernormal blood supply to the myocardium by means of a Sigmamotor pump did not increase myocardial contractile force at all (Fig. 4). Gregg et al. ${ }^{18), 19)}$ showed, however, that the increase in coronary arterial blood flow per se increases myocardial oxygen consumption in dogs, in which cardiac work was kept constant throughout the experimental period. Presently, one can not be certain of the mechanism, through which the increase in myocardial oxygen consumption apparently occurs without any change in myocardial contraction.

Wegria et al. ${ }^{13)}$ and Levy and Frankel ${ }^{21)}$ observed that the ligation of the anterior descending branch decreases cardiac output and mean systemic arterial pressure significantly. Howcver, shortly thercafter, thesc paramcters rise to or toward controls as total peripheral resistance increases. The recovery of cardiac output and systemic arterial pressure as well as the increase in total peripheral resistance are most likely caused by the reflex increase in the sympathoadrenal activity. ${ }^{22)}$ Richardson et al. ${ }^{23)}$ and Gazes et al. ${ }^{24)}$ observed that the plasma catecholamine levels increase in patients with acute myocardial infarction. In the present study, coronary artery ligation increased heart rate and myocardial contractile force of the healthy portion of the left ventricle although myocardial contractile force decreased markedly in the injured part of the ventricle. This increase in myocardial contractile force and heart rate may also indicate an evidence of the increased sympathoadrenal activity. Furthermore, in the present experiments, the intravenous administration of norepinephrine increased myocardial contractile force of the healthy portion of the ventricle, but caused essentially no change in contractile force of the injured portion. The increased myocardial contractile force is most likely caused by the direct positive inotropic action of norepinephrine and alos partially by the increased coronary blood flow which indirectly results from the rise in mean systemic arterial pressure. In contrast, no such pharmacodynamic effects were observed in the injured myocardium, since coronary artery ligation causes extreme myocardial hypoxia and thereby depletes myocardial ATP and CP. In addition, as Kattus and Gregg have reported, the coronary collateral vessels in the infarcted areas become unresponsive to norepinephrine. ${ }^{25)}$

\section{SUMMARY}

The effect of coronary artery constriction and ligation, and graded changes in coronary arterial blood flow on myocardial contractile force was studied in anesthetized open-chest dogs. The coronary constriction and stepwise decreases in coronary blood flow using a Sigmamotor pump decreased myocardial 
contractile force essentially in proportion to the decrements of the coronary flow. On the other hand, the increased coronary blood flow beyond physiological ranges did not increase contractile force. The coronary ligation decreased myocardial contractile force of the ischemic or injured areas of the ventricle, but increased the contractile force in the healthy area of the ventricle. Under these conditions, norepinephrine administration increased the contractile force of the healthy portion of the ventricle, whereas the myocardial contractile force of the injured portion remained essentially unchanged.

\section{AGknowledgements}

The author is indebted to Dr. R. O. Glinton of Sterling-Winthrop Laboratories, Rensselaer, N.Y., Dr. H. A. Strade of Organon, Inc., West Orange, N.J., and Dr. H. G. Schoepke of Abbott Laboratories, North Chicago, Ill. for generous supplies of norepinephrine (Levophed) and sodium heparin (Liquaemin and Panheparin), respectively.

\section{REFERENCES}

1. Pardee, H. E. B. : Arch. Int. Med. 26 : $244,1920$.

2. Wood, F. C. and Wolferth, G. C. : Arch. Int. Med. $47: 339,1931$.

3. Bailey, R. H., LaDue, J. S., and York, D. J. : Am. Heart J. 27 : 164, 1944.

4. Bailey, R. H., LaDue, J. S., and York, D. J. : Am. Heart J. 27 : 657, 1944.

5. Wegria, R., Segers, M., Keating, R. P., and Ward, H. P. : Am. Heart J. 38 : 90, 1949.

6. Orias, O.: Am. J. Physiol. 100:629, 1932.

7. Tennant, R. and Wiggers, G. J.: Am. J. Physiol. 112: 351, 1935.

8. Furchgott, R. F. and Lee, K. S.: Circulation 24: 416, 1961.

9. Shipley, R. E. and Wilson, C. : Proc. Soc. Exp. Biol. Med. 78 : 724, 1951.

10. Boniface, K. J., Brodie, O.J., and Walton, R. P.: Proc. Soc. Exp. Biol. Med. 84: $263,1953$.

11. Cotten, M. de V. and Bay, E. : Am. J. Physiol. 187: 122, 1956.

12. Siegel, J H. and Sonneblick, E. H.: Circulat. Res. $12: 597,1963$.

13. Wegria, R., Frank, G. W., Misrahy, G. A., Wang, H. W., Miller, R., and Case, R. B. : Am. J. Physiol. 177 : 123, 1954.

14. Szent-Györgyi, A.: The Chemistry of Muscular Contraction. New York, Academic Press, Inc., 1946.

15. Chang, I.: Quart. J. Exp. Physiol. 27: 113, 1937.

16. Michal, G., Naegle, S., Danforth, W. H., Ballard, F. B., and Bing, R. J. : Am. J. Physiol. 197: $1147,1959$.

17. Cofman, J. D. and Gregg, D. E. : Am. J. Physiol. 199: 1143, 1960.

18. Green, H. D. and Wegria, R. : Am. J. Physiol. 135 : 271, 1941-42.

19. Gregg, D. E., Rayford, C. R., Khouri, E. M., Kattus, A. A., and McKaeever, W. P. : Circultion (Abstract) $16: 888,1957$.

20. Gregg, D. E., Rayford, C. R., Khouri, E. M., Kattus, A. A. and McKeever, W. P.: Fed. Proc. 17: 61, 1958 .

21. Levy, M. N. and Frankel, A. L.: Am. J. Physiol. 172: 427, 1953.

22. Heymans, C. and Neil, E. : Reflexogenic Area of the Cardiovascular System, Little Brown, Boston, 1958.

23. Richardson, J. A., Woods, E. F., and Bagwell, E. E.: Am. J. Cardiol. 5 : 613, 1960.

24. Gazes, D. C., Richardson, J. A., and Woods, E. F.: Circulation 19:657, 1951.

25. Kattus, A. A. and Gregg, D. E. : Circulat. Res. 7:628, 1959. 